\title{
QUASAR CLUSTERING AT INTERMEDIATE REDSHIFTS
}

\author{
Michael Drinkwater \\ Institute of Astronomy, Cambridge CB30HA, England.
}

This paper summarizes recent results from a study of quasar clustering based on a survey of U.K. Schmidt objective prism plates using the Automatic Plate Measuring facility at Cambridge. New procedures have been developed to detect quasars, eliminate contamination and measure accurate redshifts from the (see Drinkwater, M.J. 1987. Mon. Not. R. astr. soc., submitted). The redshift identifications are $90 \%$ reliable with an error ( $\mathrm{rms}$ ) of $\Delta z= \pm 0.019$ for quasars to a limiting magnitude of $B_{J}=19.5$. Some 600 quasars from 6 U.K. Schmidt fields in the redshift range $1.8<z<2.6$ have been analysed for weak clustering using power spectrum analysis (Webster, A. 1976. Mon. Not. R. astr. soc. 175, 61). Plots of the statistic $Q^{\prime}$ for 2-D and 3-D (4 fields only) projections of the data are given in the figure below. The $\pm 1 \sigma$ expectation values of $Q^{\prime}$ calaulated for random simulations of the data (including non-uniform redshift selection and plate sensitivity) are indicated by vertical bars.
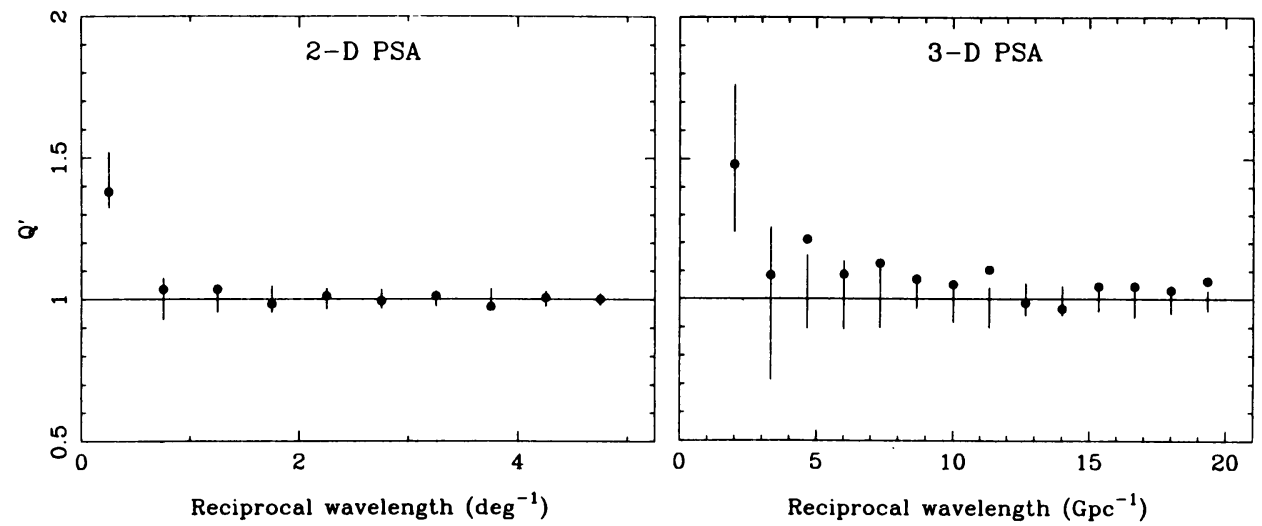

If clustering is present on a scale of $\lambda_{c}$, the value of $Q^{\prime}$ will be greater than the expectation value in the range $0<\lambda^{-1}<\lambda_{c}^{-1}$. The 2-D analysis is consistent with a random distribution, wheras some evidence for clustering on a scale of $75 \mathrm{~h}^{-1} \mathrm{Mpc}$ is evident in the 3-D case, although this represents very weak clustering (equivalent to $25 \%$ of the observed quasars lying in pairs on this scale). 